\title{
LEITER
}

\section{Condensing osteitis of the clavicle}

\section{To the Editor:}

T he article on the very rare condition of condensing osteitis of theinferomedial aspect of the clavicle ${ }^{1}$ was most interesting and prompts thefollowing short casenotes.

A 43 year old woman with a history of left chest pain was referred for radiographs of the chest and thoracic spine which demonstrated a "coin lesion" overlying the inferomedial aspect of the right clavicle. On the frontal chest radiograph the lesion appeared to be within the medial clavicle, but on the antero-posterior thoracic spine film the nodule appeared to be intrapulmonary in location due to a superimposed vessel which seemed to be part of the "coin lesion".

The dilemma was expeditiously resolved by taking radiographs specifically angled to show the medial clavicle which confirmed that the rounded sclerotic lesion was intraclavicular in location. No associated inferomedial bony spur or osteophyte was demonstrated, there was no soft tissue swelling and the sternoclavicular joint was normal.

Of particularinterestwas that the patient hadhad nopain or soft tissueswelling related to the medial aspect of the right clavicle. No history of previous trauma was elicited. Two years before the presentation of chestpain she had experienced right sided shoulder pain, and she again presented with pain in the

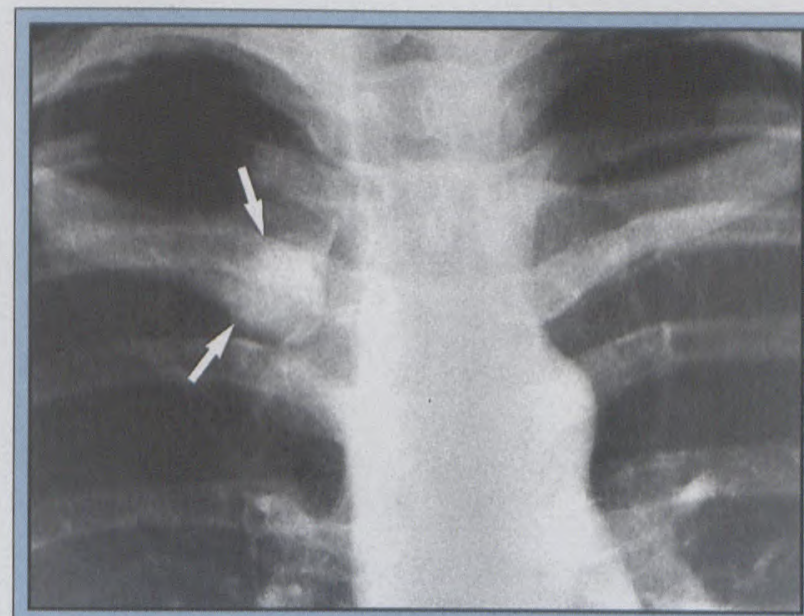

Figure 1: Close up view of right clavicle on frontal chest radiograph demonstrates rounded sclerotic area involving inferomedial aspect of right clavicle consistent with sclerosing osteitis (arrows) right shoulder two and a half years after the chest pain incident. Chest radiographs taken at this time revealed no changein appearance or size of the dense clavicular lesion.

Of 16 histologically proven cases, all occurring in women mostly presenting in the fourth or fifth decades (age range 26-63), only two were asymptomatic with thelesion discovered serendipitously ${ }^{2}$ as was the situation in the abovecase.

Condensing osteitis of the clavicle is an important condition to recognise as the plain films should be diagnostic and no further investigation should generally be required. The important condition to consider in the differential diagnosis is a solitary sclerotic metastatic deposit which would require further investigation.

Although the above patient was asymptomatic, some patients do have severe pain and non-steroidal anti-inflammatory medication may be required. In some patients where the pain is refractory, excision of the medial third of the clavicle may be necessary as a last resort. ${ }^{2}$

\section{WFC van Gelderen \\ FFRad (D) (SA) \\ ConsultantRadiologist \\ Department of Radiology \\ WangamiiBase Hospital \\ Wangami \\ NewZealand}

\section{References}

1. Ooi GC, Peh WCG. Condensing osteitis of the clavide. South African Joumal of Radiology, 1996;1[2]:25-27.

2. Greenspan A, Gerscovich E, Szabo RM, Mathews II JG. Condensing osteitis of the clavicle : a rare but frequently misdiagnosed condition. American Joumal of Roentgenology 1991;156:1011-1015.

\section{Maurice Weinbren Award in Radiology 1996}

The award is offered annually (in respect of a calendar year) by the Council of The College of Medicine of South Africa for a paper of sufficientmeritdealing either with radiodiagnosis, radiotherapy, nuclear medicine or diagnostic ultrasound.

The award consists of a Certificate anda Medal

The closing date is 15 February 1997. The Guidelines pertaining to the award can be obtained from the Executive Secretary, Mrs Bernise Bothma at 17 Milner Road, Rondebosch, 7700 or Tel:(021) 689-3161,Fax: (021) 685-3766,E-mail:cmsa-adm@iafrica.com 